\title{
A Shared-Aperture Dual-Band Planar Array With Self-Similar Printed Folded Dipoles
}

\author{
Krishna Naishadham, Senior Member, IEEE, RongLin Li, Senior Member, IEEE, Li Yang, Terrence Wu, \\ Walker Hunsicker, Associate Member, IEEE, and Manos Tentzeris, Fellow, IEEE
}

\begin{abstract}
Light-weight antenna arrays require utilizing the same antenna aperture to provide multiple functions (e.g., communications and radar) in separate frequency bands. In this paper, we present a novel antenna element design for a dual-band array, comprising interleaved printed dipoles spaced to avoid grating lobes in each band. The folded dipoles are designed to be resonant at octave-separated frequency bands (1 and $2 \mathrm{GHz}$ ), and inkjet-printed on photographic paper. Each dipole is gap-fed by voltage induced electromagnetically from a microstrip line on the other side of the substrate. This nested element configuration shows excellent corroboration between simulated and measured data, with 10-dB return loss bandwidth of at least $5 \%$ for each band and interchannel isolation better than $15 \mathrm{~dB}$. The measured element gain is 5.3 to $7 \mathrm{dBi}$ in the two bands, with cross-polarization less than $-25 \mathrm{dBi}$. A large array containing 39 printed dipoles has been fabricated on paper, with each dipole individually fed to facilitate independent beam control. Measurements on the array reveal broadside gain of 12 to $17 \mathrm{dBi}$ in each band with low cross-polarization.
\end{abstract}

Index Terms-Dual band antenna, dual frequency, inkjet printing, multifunctional antennas, nested antennas, phased array, printed dipole, shared aperture design.

\section{INTRODUCTION}

$\mathbf{I}$ $\mathrm{N}$ AEROSPACE communications, there is a pressing need to minimize the size, weight and power requirements of antenna arrays, while simultaneously implementing multiple radiation functions within the same physical aperture, a concept referred to as multifunctional arrays [1]. For example, the array may require shared aperture capability to facilitate full-duplex operation with polarization diversity, or to support multiple isolated frequency bands for communications, telemetry, radar, etc. [2]-[5]. This paper presents the design of a novel dual-band

Manuscript received January 06, 2012; revised July 19, 2012; accepted August 08,2012 . Date of publication August 31, 2012; date of current version January 30, 2013.

K. Naishadham and M. Tentzeris are with the School of Electrical and Computer Engineering, Georgia Institute of Technology, Atlanta, GA 30332 USA (e-mail: krishna.n@ieee.org; emmanouil.tentzeris@ece.gatech.edu).

R.L. Li is with the School of Electronic and Information Engineering, South China University of Technology, Guangzhou 510641, China (e-mail: lirl@scut. edu.cn).

L. Yang is with Texas Instruments, Dallas, TX 75023 USA (e-mail: lyang@gmail.com).

T. Wu was with the School of ECE, Georgia Institute of Technology, Atlanta, GA, USA. He is now with DirecTV, Los Angeles, CA 90245 USA (e-mail: wterence@gmail.com).

W. Hunsicker is with the Sensors and Electromagnetic Applications Laboratory, Georgia Tech Research Institute, Atlanta, GA 30332 USA (e-mail: walker. hunsicker@gtri.gatech.edu).

Color versions of one or more of the figures in this paper are available online at http://ieeexplore.ieee.org.

Digital Object Identifier 10.1109/TAP.2012.2216491 planar antenna array comprising interleaved folded dipoles of two different resonant sizes, but similar shapes, sharing the same physical aperture. The array is inkjet printed on photographic paper using a low-cost process [6].

Several researchers have investigated planar antennas for multiband operation, using a single feed to excite all the bands. A T-shaped planar monopole antenna with two asymmetrical horizontal strips to produce the lower and upper resonant modes is reported in [7] to cover the $2.4 / 5.2 \mathrm{GHz}$ ISM bands. The authors in [8] present the design of a planar L-shaped monopole antenna fed by a microstrip line, which utilizes parasitic coupling between the driven elements and a shorted wire to excite two resonant modes for tri-band operation. The concept of adjusting reactive coupling between parasitic and driven elements to produce dual-band operation has been used extensively in planar patch antenna geometries, including the use of flat-plate radiator above a ground plane with a shorted parasitic strip on the same face [9], printing two dissimilar coupled monopoles on either side of a substrate [10], and the use of slots and shorted pins on patches to produce modes with widely separated resonant frequencies [11], [12]. However, none of these design approaches are suitable for multiband array operation, because they are all based on single feed design, and depend on either shaping the radiating elements or adjusting parasitic coupling to produce multiband operation. A few design approaches exist for multifeed dual band antennas using stacked dielectric layers [13]-[15]. A microstrip patch antenna design with dual-band dual circular polarization is discussed in [13], where high-permittivity dielectric bars are inserted under the radiating edge of the patch in order to miniaturize the antenna and generate circularly polarized waves. Two stacked patches, one coax-fed and the other aperture-coupled, are used in [14] to produce closely spaced dual-band operation. As the two bands are tightly coupled, these designs introduce significant mutual coupling when integrated into an array. Mutual coupling between elements needs to be controlled in a shared aperture array to prevent excessive interchannel interference, and it may not be used for simultaneous tuning of the elements.

The concept of multifunctional array antennas with widely separated frequency bands, such as PCS, GSM communications bands, $\mathrm{L}, \mathrm{C}$, and $\mathrm{X}$ radar bands, sharing the same physical aperture, is a challenging problem, and has been largely unexplored [1]. The avoidance of grating lobes places an upper limit on the element spacing for each band, which in turn increases the array size for a wide scanning range. One established design approach is to utilize wideband (i.e., one band encompasses all the individual narrow bands of interest) antenna elements, such 
as Vivaldi slots, with each element connected to a wideband feed [16]. Wideband elements are not convenient for electronic scanning, because a single interelement spacing, typically implemented at the highest frequency, will not provide a symmetrical lobe structure over the entire frequency range of interest. In addition, the associated complexity of receiver design escalates considerably for wideband operation in a phased array system, requiring expensive, heavy and bulky front-ends to channelize signals from the wideband feed into multiple narrow frequency bands typically allocated for communications and radar. Alternatively, an interleaved element layout can be used to accommodate widely separated (in frequency) narrow-band channels, utilizing a different interelement spacing for each band to avoid grating lobes. A major problem in such a layout is the electromagnetic (EM) interference between channels, which can cause undesirable cross-polarization and mutual coupling.

Considerable effort has been devoted to determine an interleaved element design with minimal interchannel interference. Interleaved microstrip patches for $\mathrm{C}$-band operation and printed slots for X-band are used in [2] to form a shared aperture, dualband dual-polarization (DBDP) array. Good isolation between the bands is observed because the feed networks for the two bands are separated by two dielectric layers. The bidirectional radiation pattern of the $\mathrm{X}$-band slots necessitates a reflecting ground plane, increasing the thickness and weight of the antenna. The slots also contribute to increased cross-polarization and side-lobe levels at X-band. Instead of interleaving the elements in alternate substrates as in [2], the design in [3] utilizes a dual-band coplanar element comprising one L-band perforated patch symmetrically enclosing four C-band patches. To increase the bandwidth, stacked patches are used in both bands, with the bottom layer of patches fed by direct-coupled microstrip lines or slots, and the top layer of patches electromagnetically coupled to currents on the bottom layer patches. Considerable attention has been given to maintaining geometrical symmetry and good isolation of the feed lines, resulting in low cross-polarization over a scan range of 20 degrees in each principal plane. A variation of this design is implemented in [4] using proximity-coupled perforated microstrip patch elements at L-band, with X-band aperture coupled patches inserted below the openings of the L-band patches. However, the scan range is limited and the cross-polarization levels are relatively high. A novel design of DBDP microstrip array, with a frequency ratio of about $1: 3$, is presented in [5], wherein stacked, proximity-coupled microstrip dipoles and probe-fed square patches are used as the radiating elements at $\mathrm{S}$ - and $\mathrm{X}$-bands, respectively. The prototype array depicts measured impedance bandwidth (VSWR $\leq 2$ ) of $8.9 \%$ and $17 \%$, and cross-polarization levels of $-26 \mathrm{~dB}$ and $-31 \mathrm{~dB}$, for $\mathrm{S}$ - and $\mathrm{X}$-bands, respectively.

In this paper, we present a novel antenna design for a shared aperture dual-band array, comprising interleaved printed folded dipoles resonant at octave-separated frequency bands ( 1 and $2 \mathrm{GHz}$ ), with individual feeds for each element. Similar to the design in [3], we enforce geometrical symmetry in the array configuration for low cross-polarization and interchannel isolation, and electromagnetically couple the dipoles to feed lines printed on the other side of the substrate. However, unlike the previous design approaches for a shared aperture array [2]-[5],

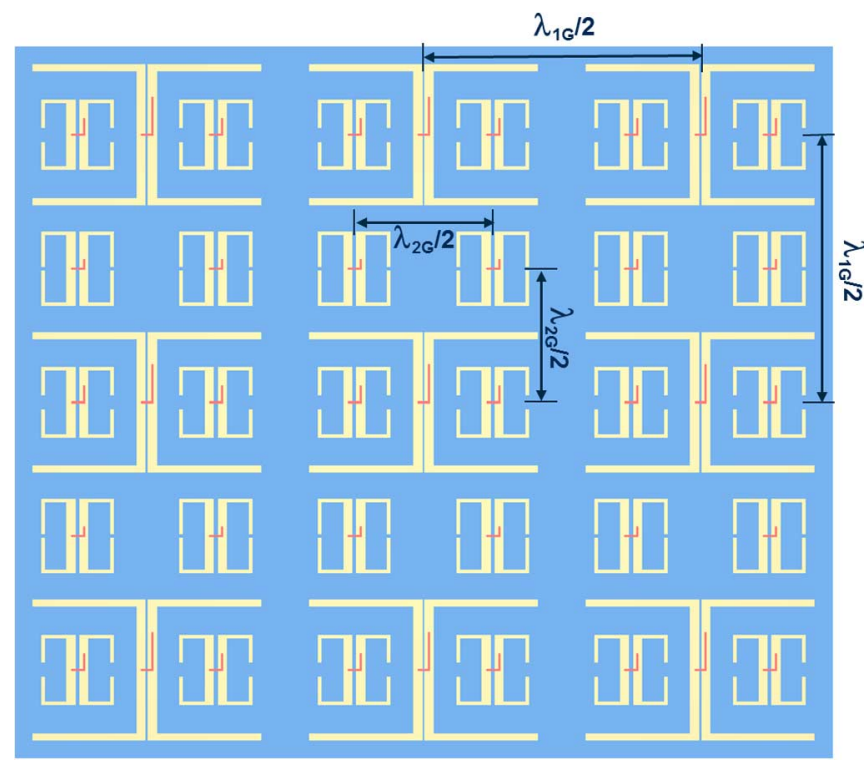

Fig. 1. Nested element dipole array with self-similar elements for dual-band operation. The wavelengths at 1 and $2 \mathrm{GHz}$ are denoted as $\lambda_{1 G}$ and $\lambda_{2 G}$, respectively.

we use self-similar antenna elements for direct scaling to other frequencies. Both the feed lines and antennas are printed on photographic paper (dielectric constant of 3.1) using low-cost ink-jet printing technology [17]-[20]. This nested element configuration has been simulated, fabricated and measured, and excellent corroboration observed between simulated and measured data. Measurements on a 39-element dual-band array ( 9 low-band and 30 high-band) printed on paper reveal broadside gain of 12-17 dBi in both bands, with cross-polarization less than $-25 \mathrm{dBi}$. This is the first practical demonstration of a large array printed on paper.

Section II introduces the layout of the dual-band array and discusses the design of self-similar nested folded dipoles. The fabrication of the antenna on photographic paper using inkjet printing is treated in Section III. Simulated and measured results of the element pattern as well as the fixed-scan broadside array pattern are discussed in Section IV, and the losses incurred by antenna fabrication on paper are addressed. The paper concludes with a brief summary in Section V.

\section{AnTEnNa Design}

The proposed antenna design involves using coplanar nested or interleaved printed dipoles to provide adequate isolation between channels, as depicted in Fig. 1 for a dual-band phased array application. The folded dipoles are assumed to be resonant at $1 \mathrm{GHz}$ and $2 \mathrm{GHz}$ for the design reported herein, but can be scaled easily due to self-similar pattern. It is noted that channel separation at integer multiples of the fundamental frequency is necessary only if self-similarity in element shape is to be preserved. In order to reduce the backlobe level and also to improve the impedance bandwidth, a ground plane is inserted $0.25 \lambda_{2 G}$ below the antenna substrate, where $\lambda_{2 G}$ is the wavelength at $2 \mathrm{GHz}$. In future design iterations, however, frequency selective surfaces will be designed to bring the ground plane closer to the antenna and reduce the overall antenna thickness. Each 


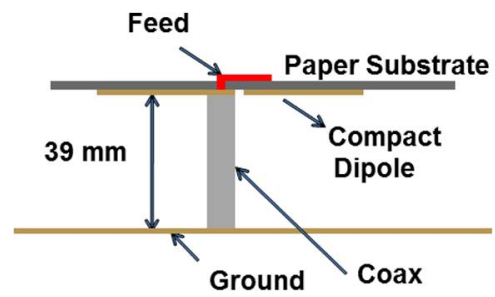

Fig. 2. Feed configuration for the dual-band element (dipole arms exaggerated in width for clarity). The ground plane is supported by a foam layer in the fabricated prototype.

dipole is gap-fed by voltage induced electromagnetically from a microstrip line on the top side of the substrate (see Fig. 2). The feed line for each antenna element also includes a series stub for independently tuning its impedance. Two high-band elements are nested inside the low-band folded dipole, one in each leg, with a spacing of half-wavelength (at $2 \mathrm{GHz}$ ) to provide grating-lobe-free operation in the array environment. In order to provide identical scan performance in the orthogonal plane, a pair of high-band elements are inserted vertically half-way between two low-band elements. Likewise, the low-band elements are spaced by half wavelength at $1 \mathrm{GHz}$, both horizontally and vertically. This self-similar dual-band element design and placement offers the capability to use the single physical aperture for multiple antenna functions, and can also be integrated with independent elements (such as patches) in stacked layers if more than two bands are desired.

Using EM simulation software (CST Microstripes-www. cst.com), the length of each dipole in the array is optimized in an infinite array environment to tune the low-band dipole to $1 \mathrm{GHz}$ and the high-band one to $2 \mathrm{GHz}$. The optimized dimensions of each dipole are shown in Fig. 3. After tuning, the mean path length of each arm of the dipole is found to be about two-third wavelength at the corresponding resonant frequency. Notice the difference in folding between the dipoles for each band, necessitated by nesting two high-band dipoles within one low-band dipole. The latter is folded only once while the former is folded twice to miniaturize within the form factor required to maintain half-wavelength spacing between the elements. The series stub on each feed line is adjustable in length for impedance matching, independently for each element at any specific array location.

The substrate has the dimensions $200 \mathrm{~mm} \times 160 \mathrm{~mm} \times$ $0.52 \mathrm{~mm}$, and a dielectric constant of 3.1. The ground plane reflector is of size $200 \mathrm{~mm} \times 160 \mathrm{~mm}$. As shown in Fig. 2, the center conductor of the coaxial feed cable is connected to the microstrip feed line, which induces by EM coupling a voltage at the gap between the two dipole arms on the other side of the substrate. One end of the cable shield is connected to an antenna arm, and the other end is terminated on the reflector. In order to minimize perturbation of antenna currents at the junction with the shield, we use SSMA connectors, with one feed per antenna. EM coupling to the dipole minimizes the feed line radiation and the associated spurious interaction between antenna elements. Since the substrate is thin, the coax-microstrip transition is predominantly capacitive and can be inductively compensated by adjusting the length of the microstrip stub. The tuning of the antenna to its resonant frequency is controlled by symmetrically adjusting the overall length of each folded arm of the dipole.

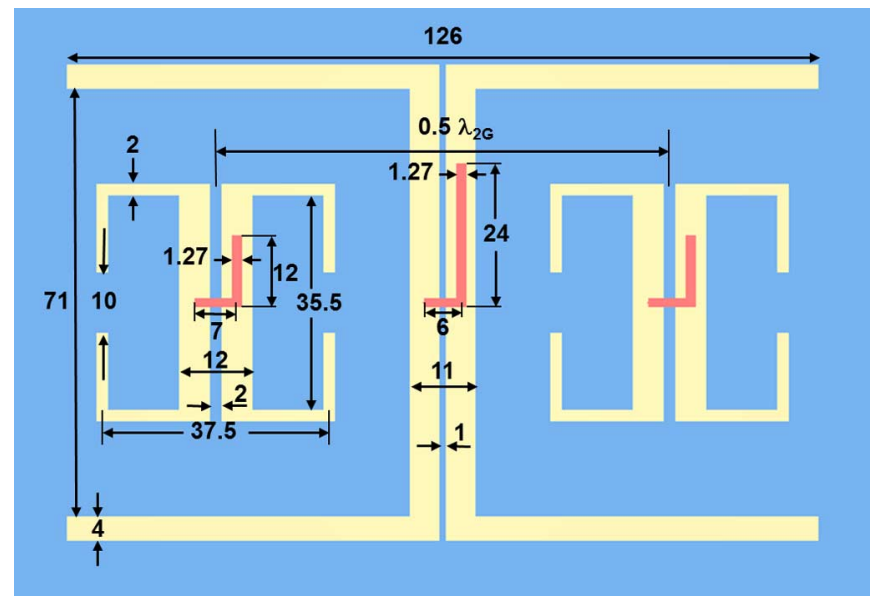

Fig. 3. Optimized dimensions (in $\mathrm{mm}$ ) of the nested dipole geometry and the feed lines. Note the scalability in design.

\section{ANTENNA FABRICATION}

In this work, we utilized inkjet printing of conductive inks to pattern the antennas on photographic paper sheets. Paper possesses a number of attributes that makes it amenable for environmentally compatible or "green" electronics. It is cellulose in nature, thus considered as a renewable resource. Additionally, it can be processed in a reel-to-reel fashion potentially enabling low-cost manufacturing solutions. Inkjet printing has been recently enhanced with the capability of printing conductive inks based on emulsions of silver nano-particles, leading to an increased deployment in printed electronics, such as flexible displays, RFIDs, sensors, solar panels, fuel cells, batteries, and antennas [17]-[20]. Inkjet printing for RF applications is a challenging endeavor, where precise control of the achieved conductivity and surface roughness of the printed trace is required.

We utilized a DMP-2800 ink-jet printer, a table-top unit available from Dimatix Inc. (www.dimatix.com), to fabricate the antennas. To ensure good RF properties of the printed device, an in-house recipe has been developed to print the antennas using Dimatix $1 \mathrm{pL}$ ink cartridges (DMC-11601) [6]. The printer head is first adjusted to achieve a print resolution of $2540 \mathrm{dpi}$, which ensures good RF conductivity up to several GHz. Cabot conductive ink CCI-300 (www.cabot-corp.com) is then jetted through the cartridges at a temperature of $40^{\circ} \mathrm{C}$, with the paper substrate maintained at $60{ }^{\circ} \mathrm{C}$. Each printed antenna is then cured in an oven for two hours at $120^{\circ} \mathrm{C}$.

\section{RESUlts AND Discussion}

\section{A. Nested Element Measurements}

The dual-band antenna has been designed using the commercial time-domain simulator, CST Microstripes, and optimized for good match (VSWR better than 1.5) at $1 \mathrm{GHz}$ and $2 \mathrm{GHz}$. Antenna losses are not considered in the simulation. The optimized antenna (see Fig. 3) has been fabricated and its radiation pattern, as well as S-parameters, have been characterized experimentally. Fig. 4 shows the antenna trace comprising the dual-band elements printed on photographic paper substrate. For clarity, the feed configuration and the ground plane are not shown. 


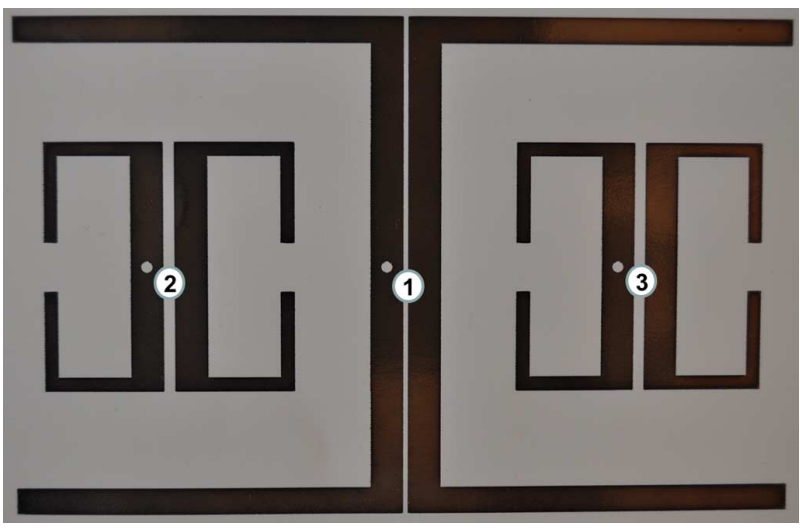

Fig. 4. Fabricated nested antenna element.

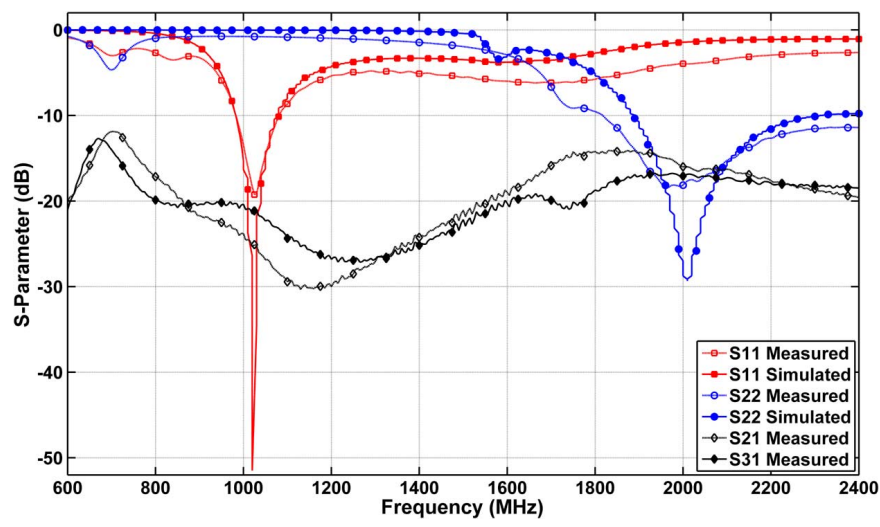

Fig. 5. Comparison between measured and simulated scattering parameters.

The comparison of simulated and measured scattering parameters is depicted in Fig. 5. The antenna elements are numbered as indicated in Fig. 4. Excellent agreement is observed between the two sets of data, with the measured return loss of the low-band $(1 \mathrm{GHz})$ at $19 \mathrm{~dB}$ and that of the high-band $(2 \mathrm{GHz})$ at $17 \mathrm{~dB}$. Because the ground plane is located at a depth of quarter wavelength (at $2 \mathrm{GHz}$ ) below the substrate, the bandwidth of the high-band is larger than that of the low-band. The measured return loss of the two symmetrical high-band elements show similar variation with frequency, and only S22 is plotted. The measured isolation between the low-band element and either of the two high-band elements is observed to be around $-17 \mathrm{~dB}$ around $2 \mathrm{GHz}$, and $-25 \mathrm{~dB}$ at $1 \mathrm{GHz}$. Therefore, it is anticipated that the interference between the two bands in an array environment will not be significant.

Radiation pattern of the antenna has been measured in an anechoic chamber at Georgia Tech Research Institute. Fig. 6 compares the simulated and measured E-plane patterns at $1 \mathrm{GHz}$. The measured peak gain is $7 \mathrm{dBi}$ whereas the simulated directivity is $8.2 \mathrm{dBi}$. Considering the losses in the element and the systematic as well as random measurement errors attributed to chamber and test equipment, which are collectively estimated as 0.5 to $1 \mathrm{~dB}$, it is evident that the simulated and measured gain values are in good agreement. The measured cross-polarization is better than $-30 \mathrm{~dB}$ relative to the main beam level. In general, the absolute measured cross-polarization levels are higher than the simulated levels because losses are not considered in

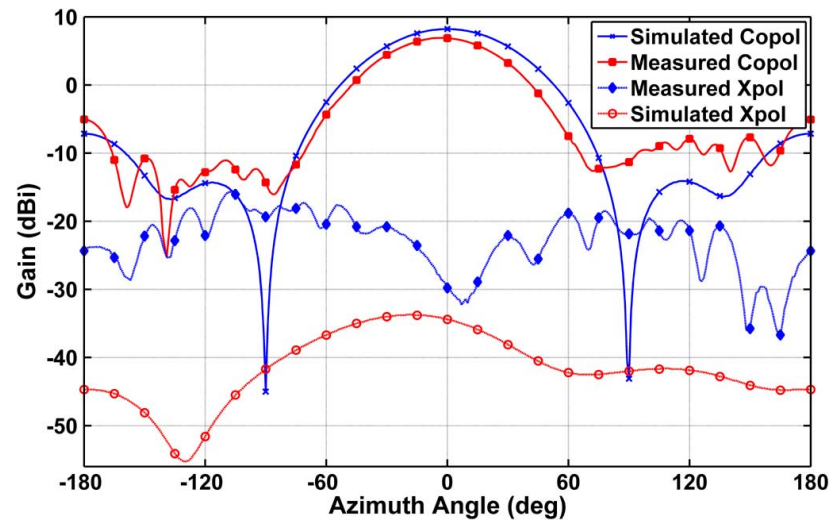

Fig. 6. Comparison of simulated and measured E-plane patterns at $1 \mathrm{GHz}$.

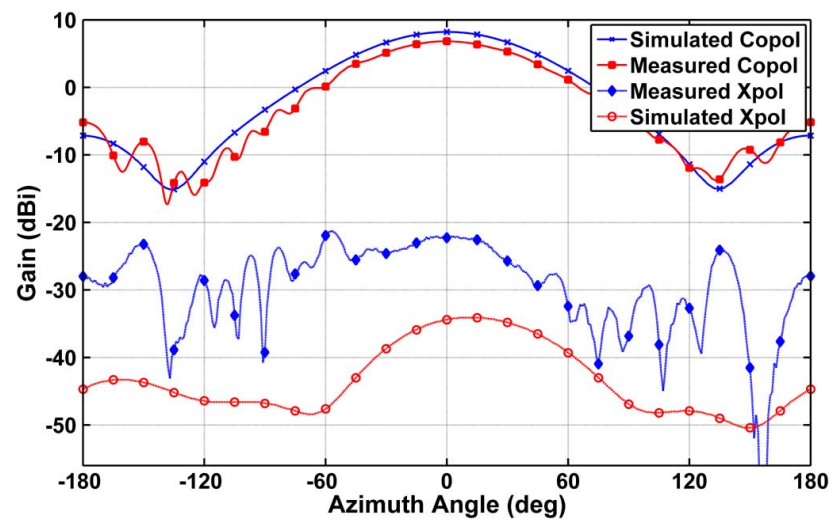

Fig. 7. Comparison of simulated and measured $\mathrm{H}$-plane patterns at $1 \mathrm{GHz}$.

the simulation, and the measurement accuracy degrades at the lower gain amplitudes.

Fig. 7 compares the simulated and measured H-plane patterns at $1 \mathrm{GHz}$. The measured peak gain is $6.9 \mathrm{dBi}$ whereas the simulated directivity is $8.2 \mathrm{dBi}$. Considering the losses in the antenna and the measurement errors, it is evident that the simulated and measured gain values are in reasonable agreement. The beamwidth is broader for the H-plane pattern than the E-plane pattern. This is typical of printed dipoles because of the uneven influence of ground plane currents in the two principal planes. The measured cross-polarization over the extent of the main beam is higher in the H-plane, but still lower than $-25 \mathrm{~dB}$ relative to the beam maximum. In summary, the agreement between the simulated and measured patterns in both planes is within the measurement accuracy.

Fig. 8 compares the simulated and measured E-plane patterns of Element 2 at $2 \mathrm{GHz}$. The pattern is asymmetric for both sets of data because of mutual coupling with the other high-band element located half wavelength away along the E-plane. A similar result, showing asymmetry in the E-plane pattern, has been observed for Element 3. The mutual coupling between the $2 \mathrm{GHz}$ dipoles is higher in the E-plane than in the H-plane, and therefore, the pattern is much less asymmetric in the H-plane (see Fig. 9). The measured peak gain (see Fig. 8) is $6 \mathrm{dBi}$ whereas the simulated directivity is $7.8 \mathrm{dBi}$. This discrepancy is larger than that in the low-band pattern in Fig. 6, because of the higher metallization and dielectric losses at $2 \mathrm{GHz}$. Nonetheless, the 


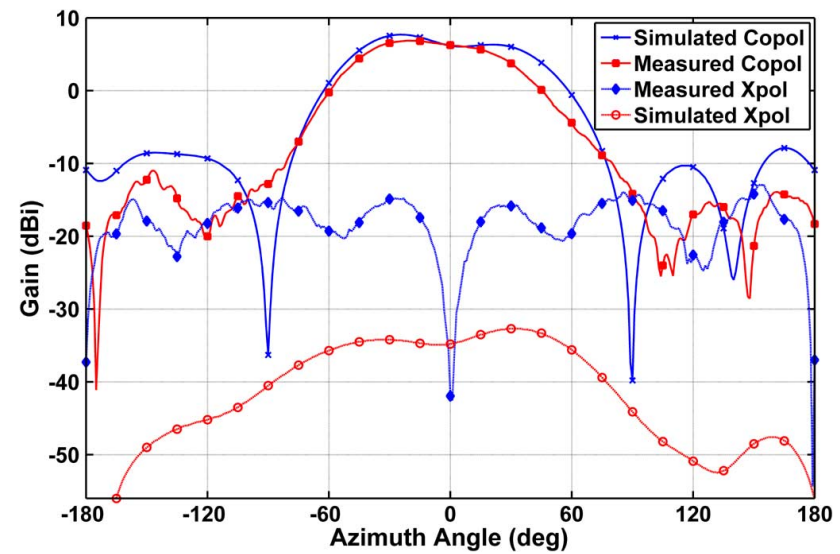

Fig. 8. Comparison of simulated and measured E-plane patterns at $2 \mathrm{GHz}$ for Element 2 (Element 3 terminated).

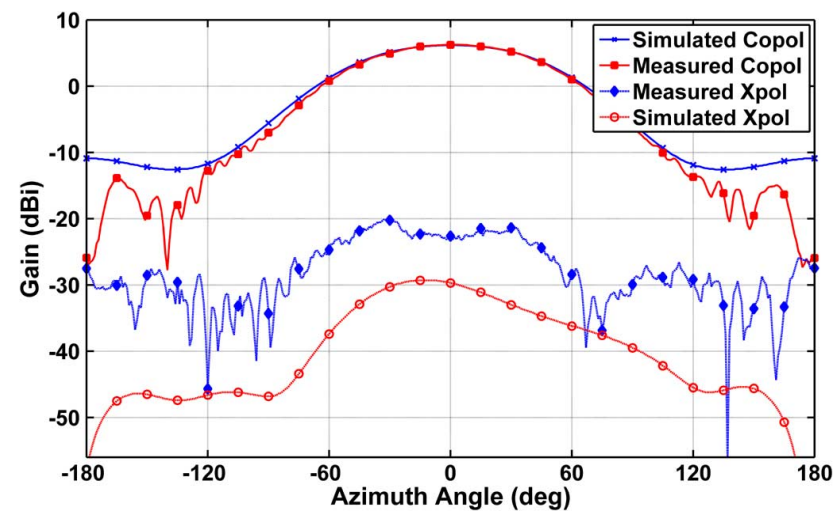

Fig. 9. Comparison of simulated and measured $\mathrm{H}$-plane patterns at $2 \mathrm{GHz}$ for Element 2 (Element 3 terminated).

agreement between measured and simulated radiation pattern data is gratifying given that the two high-band elements are separated by only a half wavelength, and are completely interleaved within the low-band element.

Fig. 9 compares the simulated and measured $\mathrm{H}$-plane patterns of Element 2 at $2 \mathrm{GHz}$. A similar result (not plotted) has been observed for Element 3 . The measured cross-polarization ratio is better than $-25 \mathrm{dBi}$ and is considerably better than that in the E-plane due to lower mutual coupling. The measured peak gain is $5.3 \mathrm{dBi}$ whereas the simulated directivity is $6.2 \mathrm{dBi}$. The lower cross-polarization at $2 \mathrm{GHz}$ results in good agreement between the two sets of copol data.

\section{B. Array Radiation Pattern}

The array layout shown in Fig. 1 has been fabricated by ink-jet printing the pattern on $3^{\prime \prime} \times 4^{\prime \prime}$ sections of paper and joining them using Kapton tape. The fabricated array, shown in Fig. 10, consists of 39 elements (9 1-GHz elements, 30 nested 2-GHz elements) mounted on $39 \mathrm{~mm}$ thick foam layer backed by an aluminum ground plane. The wooden support is only for mechanical interface with the antenna positioner. The dipole antenna elements are on the back side of the (white) paper substrate, and the microstrip feed lines, numbered sequentially per the corresponding radiators, are shown on the front side. Coaxial connections are made using room-temperature conductive silver epoxy (H20E from Ted Pella, Inc., Catalog No.

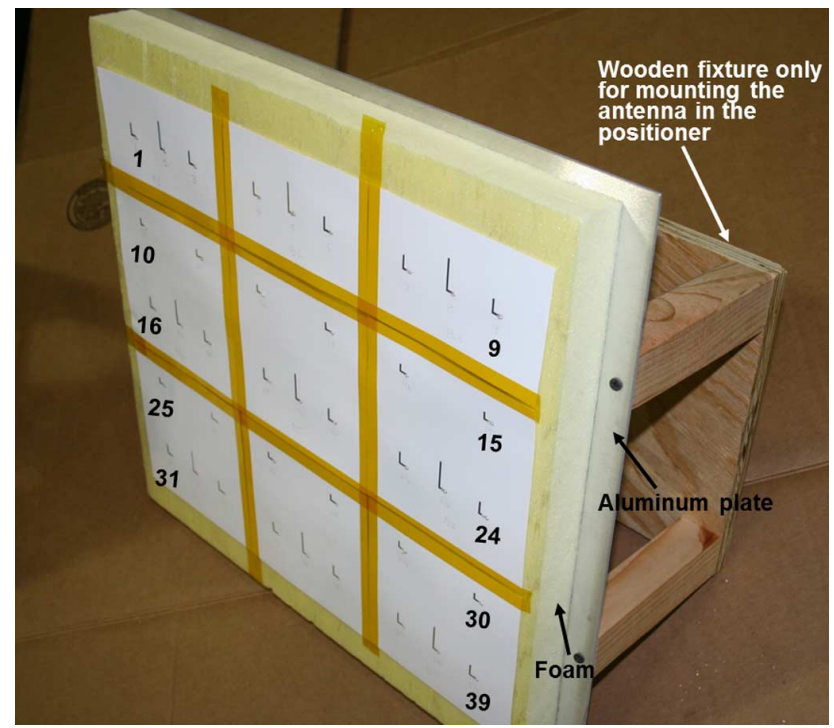

Fig. 10. Fabricated dual-band array.

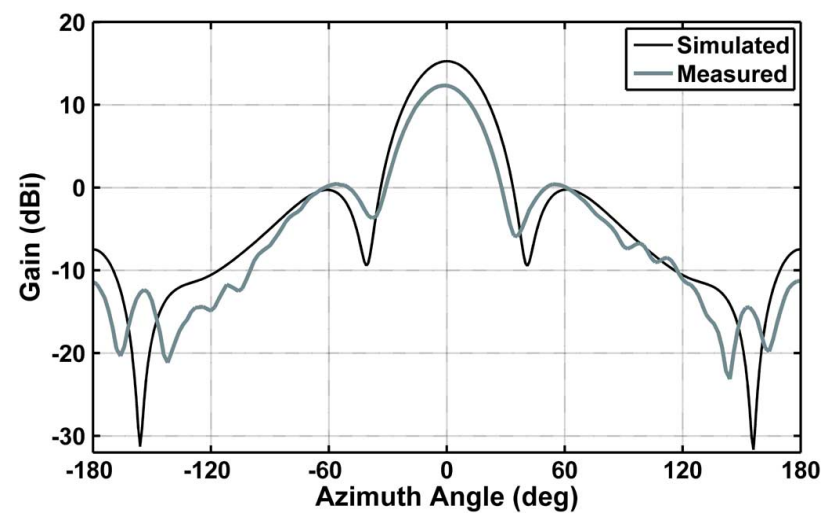

Fig. 11. Comparison of simulated and measured $\mathrm{H}$-plane patterns for the $1 \mathrm{GHz}$ array (9 elements).

16014), with the shield terminated on a dipole arm and the center conductor on the microstrip line (see Fig. 2). In order to eliminate parallel plate modes, one end of the coaxial shield is connected to the ground plane. Silver epoxy has considerable conductive loss at microwave frequencies compared to solder, and thus will affect the radiation efficiency of the array. In addition, epoxy contacts to the antenna elements can be brittle, causing potential disruption in current flow and affecting the radiation pattern.

The gain of the array has been measured in an anechoic chamber, exciting each $1 \mathrm{GHz}$ element separately. The thirty high-band elements are measured in interleaved pairs at $2 \mathrm{GHz}$ using Narda 4372-2 $3 \mathrm{~dB}$ power dividers. The elements which are not excited are terminated in $50 \Omega$. All the elements are assumed to be fed with equal amplitude and constant phase. The measured gain patterns of all the elements are coherently added to compute the broadside array pattern.

Fig. 11 compares the measured and simulated H-plane gain patterns for the $1 \mathrm{GHz}$ array. Reasonable agreement is observed for the main lobe amplitude and beamwidth, with peak measured gain of $12 \mathrm{dBi}$ and peak simulated directivity of $15.3 \mathrm{dBi}$. The discrepancy in peak gain is attributed to the fact that losses in 


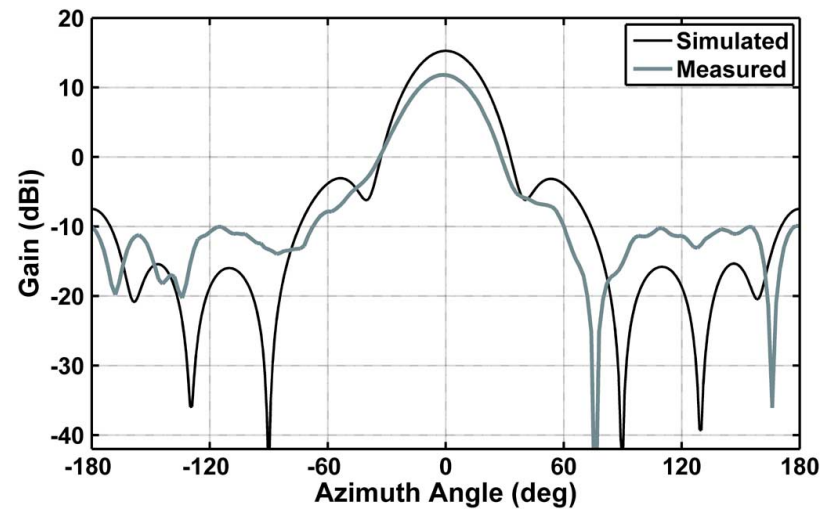

Fig. 12. Comparison of simulated and measured E-plane patterns for the $1 \mathrm{GHz}$ array (9 elements).

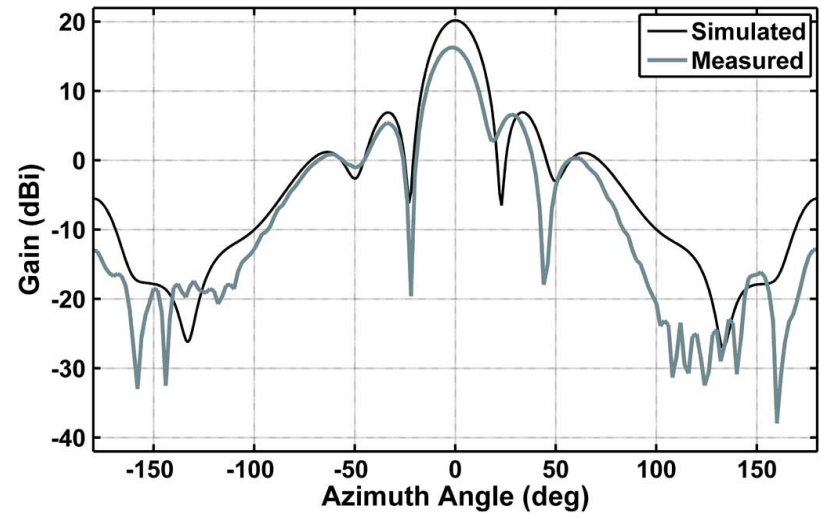

Fig. 13. Comparison of simulated and measured $\mathrm{H}$-plane patterns for the $2 \mathrm{GHz}$ array (30 elements).

the antenna, comprising silver epoxy ohmic loss, substrate loss, and the mismatch loss due to slight detuning of the fabricated array, are not accounted in the simulation. Gain measurement error on the large array has been estimated at $\pm 1 \mathrm{~dB}$. Fig. 12 plots the measured and simulated gain patterns for the $1 \mathrm{GHz}$ array in the E-plane. A similar agreement as in the H-plane pattern is observed, with the simulated results tracking the measurements closely after taking into account the estimated antenna losses. The cross-polarization in each case is measured to be lower than $-25 \mathrm{~dB}$.

Before we performed pattern measurements on the $2 \mathrm{GHz}$ array, we measured the frequency-dependent insertion loss of Narda 4372-2 $3 \mathrm{~dB}$ power divider used in data collection. The frequency-averaged insertion loss over $200 \mathrm{MHz}$ span, measured at the two output ports, is found to be $0.76 \mathrm{~dB}$. This loss is added to the measured element pattern before coherent array summation to compute the broadside fixed scan pattern.

Fig. 13 compares the measured and simulated H-plane gain patterns for the $2 \mathrm{GHz}$ array, comprising 30 dipoles nested within 9 low-band dipoles (see Fig. 1). Reasonable agreement is observed for the main lobe amplitude and beamwidth, with peak measured gain of $16.2 \mathrm{dBi}$ and peak simulated directivity of $20.2 \mathrm{dBi}$. This discrepancy of $4 \mathrm{~dB}$ is attributed to higher conductive and dielectric losses at $2 \mathrm{GHz}$ compared to $1 \mathrm{GHz}$, as well as mismatch loss due to detuning, which are not accounted in the simulation.

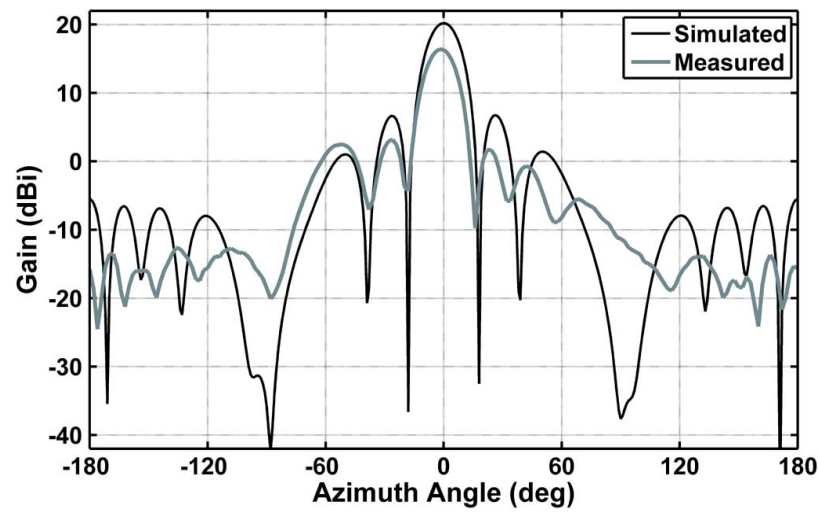

Fig. 14. Comparison of simulated and measured E-plane patterns for the $2 \mathrm{GHz}$ array (30 elements).

Fig. 14 compares the measured and simulated gain patterns for the $2 \mathrm{GHz}$ array in the E-plane, showing a similar agreement as in the H-plane pattern. The cross-polarization in each case (not shown for clarity) is about $-26 \mathrm{~dB}$ for the H-plane pattern and $-22 \mathrm{~dB}$ for the E-plane pattern.

\section{Conclusion}

We have presented a novel self-similar antenna design for a dual-band phased array configuration, comprising nested printed dipoles with individual feeds to independently control the element amplitude and phase in each band for adaptive beam-forming and MIMO applications. The folded dipoles are resonant at octave-separated frequency bands $(1 \mathrm{GHz}$ and $2 \mathrm{GHz}$ ), and fabricated on paper using eco-friendly, low-cost ink-jet printing technology. Each dipole is gap-fed in an unbalanced mode by voltage induced electromagnetically from a microstrip line on the other side of the substrate. The feed line includes a stub for improving the impedance match. This nested element configuration has been simulated, fabricated and measured, and excellent corroboration observed between simulated and measured data. The return loss shows well-centered dual bands with 10-dB bandwidth of at least $5 \%$ for each, and measured interchannel isolation better than $15 \mathrm{~dB}$. The measured copolarized element gain is about 5.3 to $7 \mathrm{dBi}$ in the two principal planes, with cross-polarization less than $-25 \mathrm{dBi}$ in the H-plane, and slightly higher in the E-plane. It has been shown that shielding provided by the interleaved design reduces coupling between the high-band elements in the H-plane. The light-weight nested antenna elements have been integrated into the first fully functional dual-band array on paper substrate. Without the $39 \mathrm{~mm}$ spacer below the substrate to minimize the backlobe, the 39-element antenna array weighs less than 500 grams, and is suitable for conformal installation on unmanned aerial vehicles. The peak gain of the array at broadside has been measured to be approximately $12 \mathrm{dBi}$ and $16 \mathrm{dBi}$ at $1 \mathrm{GHz}$ and $2 \mathrm{GHz}$, respectively, with cross-polarization better than $-25 \mathrm{~dB}$.

It is worth noting that paper substrate has higher dielectric loss (loss tangent $\sim 0.07$ at $2 \mathrm{GHz}$ ) than typical flexible substrates used in antenna design, such as liquid crystal polymer. Furthermore, conductive silver epoxy connections to metallization pattern on paper, necessitated by preclusion of solder due to its high melting point, are relatively brittle and contribute significant loss in a moderately large array. Thus, the advantages 
of inkjet printing on paper are offset by several limitations: (a) relatively high conductive and dielectric losses, (b) vias and plated-through-holes cannot be reliably fabricated using inkjet printing, and (c) considerable mechanical warping that we observed on a relatively small array precludes paper as an antenna substrate in array applications. Nonetheless, printing antennas on paper makes it easier to develop prototypes compared to wet etching.

We are investigating high impedance periodic surfaces for the ground plane to reduce the antenna thickness. Another possibility for a thinner antenna is to use slot dipoles [21] and slot loops [22] instead of printed strip dipoles, which also results in better isolation between the bands and lower cross-polarization. The ground plane below a printed slot element can be brought closer to the radiator by using an electromagnetically coupled stripline feed, which minimizes feed line radiation significantly [23].

\section{REFERENCES}

[1] "Multifunction antennas and antenna systems," IEEE Trans. Antennas Propag., vol. 54, no. 1, Jan. 2006.

[2] R. Pokuls, J. Uher, and D. M. Pozar, "Dual-frequency and dual-polarization microstrip antennas for SAR applications," IEEE Trans. Antennas Propag., vol. 46, no. 9, pp. 1289-1296, Sep. 1998.

[3] L. L. Shafai, W. A. Chamma, M. Barakat, P. C. Strickland, and G. Séguin, "Dual-band dual-polarized perforated microstrip antennas for SAR applications," IEEE Trans. Antennas Propag., vol. 48, no. 1, pp. 58-66, Jan. 2000.

[4] D. M. Pozar and S. D. Targonski, A Shared-Aperture Dual-Band DualPolarized Microstrip Array, vol. 49, pp. 150-157, Feb. 2001.

[5] X. Qu, S. S. Zhong, Y. M. Zhang, and W. Wang, "Design of an S/X dualband dual-polarised microstrip antenna array for SAR applications," IET Microw. Antennas Propag., vol. 1, no. 2, pp. 513-517, Apr. 2007.

[6] A. Rida, L. Yang, R. Vyas, and M. M. Tentzeris, "Conductive inkjetprinted antennas on flexible low-cost paper-based substrates for RFID and WSN applications," IEEE Antennas Propagat. Mag., vol. 51, no. 3, pp. 13-23, Jun. 2009.

[7] Y.-L. Kuo and K.-L. Wong, "Printed double-T monopole antenna for 2.4/5.2 GHz dual-band WLAN operations," IEEE Trans. Antennas Propag., vol. 51, no. 9, pp. 2187-2192, Sep. 2003.

[8] J.-Y. Jan and L.-C. Tseng, "Small planar monopole antenna with a shorted parasitic inverted-L wire for wireless communications in the 2.4, 5.2, and 5.8-GHz bands," IEEE Trans. Antennas Propag., vol. 52, no. 7, pp. 1903-1905, Jul. 2004.

[9] K.-L. Wong, L.-C. Chou, and C.-M. Su, "Dual-band flat-plate antenna with a shorted parasitic element for laptop applications," IEEE Trans. Antennas Propag., vol. 53, no. 1, pp. 539-544, Jan. 2005.

[10] R. L. Li, B. Pan, J. Laskar, and M. M. Tentzeris, "A novel low-profile broadband dual-frequency planar antenna for wireless handsets," IEEE Trans. Antennas Propag., vol. 56, no. 4, pp. 1155-1162, Apr. 2008.

[11] B. F. Wang and Y. T. Lo, "Microstrip antennas for dual-frequency operation," IEEE Trans. Antennas Propag., vol. 32, no. 9, pp. 938-943, Sep. 1984.

[12] J.-H. Lu, "Broadband dual-frequency operation of circular patch antennas and arrays with a pair of L-shaped slots," IEEE Trans. Antennas Propag., vol. 51, no. 5, pp. 1018-1023, May 2003.

[13] B. Lee, F. J. Harackiewicz, K.-H. Kong, J. Byun, and S.-H. Yang, "Dual-band dual-circularly polarized sector antenna for GPS and DAB systems," Microw. Opt. Tech. Lett., vol. 46, no. 1, pp. 43-46, Jul. 2005.

[14] J.-F. Zürcher, A. Skrivervik, O. Staub, and S. Vaccaro, "A compact dual port, dual frequency printed antenna with high decoupling," Microw. Opt. Tech. Lett., vol. 19, no. 2, pp. 131-137, Oct. 1998.

[15] P. Li, K. M. Luk, and K. L. Lau, "A dual-feed dual-band L-probe patch antenna," IEEE Trans. Antennas Propag., vol. 53, no. 7, pp. 2321-2323, Jul. 2005.

[16] T.-Y. Yun, C. Wang, P. Zepeda, C. T. Rodenbeck, M. R. Coutant, M. Li, and $\mathrm{K}$. Chang, "A 1-21 GHz low-cost, multi-frequency and full-duplex phased array antenna system," IEEE Trans. Antennas Propag., vol. 50, no. 5, pp. 641-650, May 2002.

[17] D. C. Thompson, O. Tantot, H. Jallageas, G. E. Ponchak, M. M Tentzeris, and J. Papapolymerou, "Characterization of liquid crystal polymer material and transmission lines on LCP substrates from 30 to $110 \mathrm{GHz}$," IEEE Trans. Microw. Theory Tech., vol. 52, no. 4, pp. 1343-1352, Apr. 2004.
[18] L. Yang, A. Rida, R. Vyas, and M. M. Tentzeris, "RFID Tag and RF structures on a paper substrate using inkjet-printing technology," IEEE Trans. Microw. Theory Tech., vol. 55, no. 12, pp. 2894-2901, Dec. 2007.

[19] M. Berggren, T. Kugler, T. Remonen, D. Nilsson, M. Chen, and P. Norberg, "Paper electronics and electronic paper," in Proc. IEEE Polymers Adhesives Microelectron. Photon. Conf., Oct. 2001, pp. 300-303.

[20] B. Farrell and M. St. Lawrence, "The processing of liquid crystalline polymer printed circuits," in Proc. IEEE Electron. Components Technol. Conf., May 2002, pp. 667-671.

[21] R. Hasse, K. Naishadham, W. H. Hunsicker, M. M. Tentzeris, and T. $\mathrm{Wu}$, "Full wave analysis of a dual-frequency printed slot antenna with a microstrip feed," presented at the IEEE Antennas Propag. Symp., Toronto, ON, Canada, Jul. 2010.

[22] W. Hunsicker, K. Naishadham, and R. Hasse, "Integration of an $\mathrm{X}$-band microstrip patch array and beamformer for a multifunctional antenna array," presented at the IEEE Phased Array Syst. Technol. Symp., Boston, MA, Oct. 2010

[23] K. Hirose and H. Nakano, "Dual loop slot antenna with simple feed," Electron. Lett., vol. 25, no. 18, pp. 1218-1219, 1989.

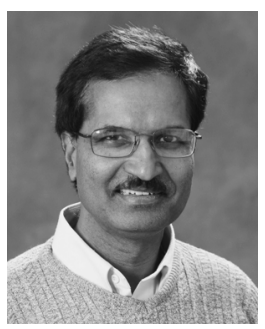

Krishna Naishadham (S'84-M'86-SM'97) received the M.S. degree from Syracuse University, Syracuse, NY, and the Ph.D. degree from the University of Mississippi, Oxford, both in electrical engineering, in 1982 and 1987, respectively.

He served on the faculty of Electrical Engineering for 15 years at the University of Kentucky and Wright State University (tenured Professor). From 2002-2008, he was a Research Scientist at Massachusetts Institute of Technology Lincoln Laboratory, where he contributed innovative hybrid asymptotic techniques and spectral estimation methods for EM signature analysis and target feature extraction. In 2008, he joined Georgia Institute of Technology, where he currently performs research on applied EM, RF nanotechnology and novel multifunctional antenna design. He published four book chapters and over 150 papers in professional journals and conference proceedings.

Dr. Naishadham serves as an Associate editor of the International Journal of Microwave Science and Technology. He is Chair of the Joint IEEE AP/MTT Chapter at Atlanta and serves on the Technical Program Committee for the International Microwave Symposium.

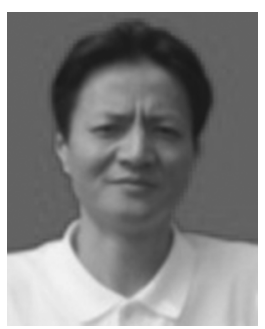

RongLin Li, (M'02-SM'03) received the B.S degree in electrical engineering from Xi' an Jiaotong University, China, in 1983, and the M.S. and Ph.D. degrees in electrical engineering from Chongqing University, China, in 1990 and 1994, respectively.

From 1983 to 1987, he worked as an Assistan Electrical Engineer in Yunnan Electric Power Research Institute. From 1994 to 1996, he was a Postdoctoral Research Fellow in Zhejiang University, China. In 1997, he visited Hosei University, Japan, as an HIF (Hosei International Fund) Research Fellow. In 1998, he became a Professor in Zhejiang University. In 1999, he visited the University of Utah, as a Research Associate. In 2000, he worked as a Research Fellow at the Queen's University of Belfast, UK. Since 2001, he has been a Research Scientist with Georgia Institute of Technology, Atlanta. Currently, he is an Endowed Professor in the South China University of Technology. He has published more than 100 papers in refereed journals and conference proceedings, and 3 book chapters. His current research interests include new design techniques for antennas in mobile and satellite communication systems, phased arrays and smart antennas for radar applications, wireless sensors and RFID technology, electromagnetics and information theory.

Dr. Li is a member of the IEEE International Compumag Society. He currently serves as an Editor of the ETRI Journal and a reviewer for a number of international journals, including the IEEE TRANSACTIONS ON ANTENNAS and Propagation, IEEE Antennas and Wireless Propagation Letters, IEEE Microwave and Wireless Components Letters, IET Microwave, Antennas \& Propagation, Progress in Electromagnetic Research, Journal of Electromagnetic Waves and Applications, and the International Journal of Wireless Personal Communications. He was a member of the Technical Program Committee for IEEE-IMS 2008-2012 Symposia and a session chair for several IEEE-APS Symposia. He was the recipient of the 2009 Georgia Tech-ECE Research Spotlight Award. 
Li Yang, photograph and biography not available at the time of publication.

Terrence $\mathbf{W u}$, photograph and biography not available at the time of publication.

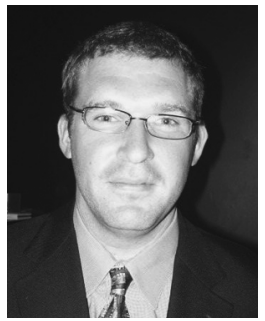

Walker Hunsicker (A'10) was born in Tupelo, MS, in 1978. He received the B.S.E.E. degree from the University of Mississippi, Oxford, in 2000, and the Master's degree in engineering science (electromagnetics), in 2007 . He is currently working toward the $\mathrm{Ph} . \mathrm{D}$. degree in electrical engineering at the University of Mississippi with expected graduation in 2013.

In 2002, he worked as a Research Engineer at the Naval Air Station, Patuxent River, MD, where he performed analysis on spatial power combiners using the method of moments. In 2004, he joined Radiance Technologies, Oxford, Mississippi, as an antenna design engineer to investigate next generation missile defense array technologies. From 2009, he has been with the Sensors and Electromagnetic Applications Laboratory at Georgia Tech Research Institute (GTRI), and specializes in antenna measurement and the design of low profile scanned arrays.

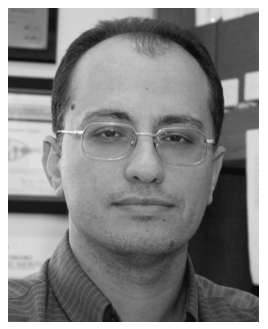

Manos Tentzeris (S'89-M'92-SM'03-F'10) received the Diploma degree (magna cum laude) in electrical and computer engineering from the National Technical University of Athens, Athens, Greece, and the M.S. and Ph.D. degrees in electrical engineering and computer science from the University of Michigan, Ann Arbor.

$\mathrm{He}$ is currently a Professor with School of Electrical and Computer Engineering, Georgia Institute of Technology, Atlanta. He has published more than 370 papers in refereed journals and conference proceedings, five books and 19 book chapters. He has helped develop academic programs in highly integrated/multilayer packaging for $\mathrm{RF}$ and wireless applications using ceramic and organic flexible materials, paper-based RFIDs and sen- sors, biosensors, wearable electronics, inkjet-printed electronics, "Green" electronics and power scavenging, nanotechnology applications in RF, microwave MEMs, SOP-integrated (UWB, multiband, mmW, conformal) antennas, and adaptive numerical electromagnetics (FDTD, MultiResolution Algorithms) and heads the ATHENA research group (20 researchers). He served as the Georgia Electronic Design Center Associate Director for RFID/Sensors research from 2006-2010 and as the Georgia Tech NSF-Packaging Research Center Associate Director for RF Research and the RF Alliance Leader from 2003-2006. He was a Visiting Professor with the Technical University of Munich, Germany for the summer of 2002, a Visiting Professor with GTRI-Ireland in Athlone, Ireland for the summer of 2009 and a Visiting Professor with LAAS-CNRS in Toulouse, France for the summer of 2010 . He has given more than 100 invited talks to various universities and companies all over the world.

Dr. Tentzeris is a member of URSI-Commission D, a member of the MTT-15 committee, an Associate Member of EuMA, a Fellow of the Electromagnetic Academy, and a member of the Technical Chamber of Greece. $\mathrm{He}$ is one of the IEEE MTT-S Distinguished Microwave Lecturers from 2010-2012. He was the recipient/corecipient of the 2010 IEEE Antennas and Propagation Society Piergiorgio L. E. Uslenghi Letters Prize Paper Award, the 2010 Georgia Tech Senior Faculty Outstanding Undergraduate Research Mentor Award, the 2009 IEEE TRANSACTIONS ON COMPONENTS and Packaging Technologies Best Paper Award, the 2009 E. T. S. Walton Award from the Irish Science Foundation, the 2007 IEEE APS Symposium Best Student Paper Award, the 2007 IEEE IMS Third Best Student Paper Award, the 2007 ISAP 2007 Poster Presentation Award, the 2006 IEEE MTT Outstanding Young Engineer Award, the 2006 Asian-Pacific Microwave Conference Award, the 2004 IEEE TRANSACTIONS ON ADVANCED PACKAGING Commendable Paper Award, the 2003 NASA Godfrey "Art" Anzic Collaborative Distinguished Publication Award, the 2003 IBC International Educator of the Year Award, the 2003 IEEE CPMT Outstanding Young Engineer Award, the 2002 International Conference on Microwave and Millimeter-Wave Technology Best Paper Award (Beijing, China), the 2002 Georgia Tech-ECE Outstanding Junior Faculty Award, the 2001 ACES Conference Best Paper Award and the 2000 NSF CAREER Award and the 1997 Best Paper Award of the International Hybrid Microelectronics and Packaging Society. He was the TPC Chair for IEEE IMS 2008 Symposium and the Chair of the 2005 IEEE CEM-TD Workshop and he is the Vice-Chair of the RF Technical Committee (TC16) of the IEEE CPMT Society. He is the founder and chair of the RFID Technical Committee (TC24) of the IEEE MTT Society and the Secretary/Treasurer of the IEEE C-RFID. He is an Associate Editor of IEEE TRANSACTIONS ON MicrowaVE THEORY and TeChNiques, IEEE TRANSACtions On AdVAnCED PACKAgIng, and the International Journal on Antennas and Propagation. 